\title{
Valoración social de los sistemas agrarios periurbanos. Aplicación al sistema periurbano de la Huerta de Valencia
}

\author{
Inmaculada Marqués Pérez y Baldomero Segura García del Río \\ Departamento de Economía y Ciencias Sociales. Universitat Politècnica de \\ Valènciaimarques@esp.upv.es, bsegura@upvnet.upv.es
}

\begin{abstract}
Resumen. El debate en torno a la multifuncionalidad de los sistemas agrarios, las reformulaciones más recientes del concepto y sus conexiones con las políticas territoriales, así como los corolarios más interesantes que resultan del mismo, constituyen la base de un enfoque muy apropiado para legitimar la intervención pública, en aras de la conservación de sistemas agrarios a los que se reconoce la provisión de ciertos bienes y servicios. Sin embargo, la dificultad de esta intervención pública tiene su mayor obstáculo en la determinación de las preferencias de la población por estos bienes y servicios que presta la agricultura por tratarse de bienes públicos sin mercado. Las técnicas de análisis multicriterio como Analytic Hierarchy Process (AHP) puede ayudar a valorar las prioridades de los distintos colectivos sociales afectados mediante el análisis de la importancia relativa que el conjunto de la sociedad otorga a las diferentes funciones. Los resultados pueden integrarse en las decisiones de política que adopten las autoridades que llevarán a la oferta de bienes públicos demandados por la sociedad.
\end{abstract}

Palabras clave: Agricultura multifuncional, bienes públicos, producción conjunta, externalidades, fallo de mercado, óptimo social, multicriterio.

\begin{abstract}
The debate on the multifunctionality of agriculture and its connections with territorial policies are the basis of the most appropriate approach to legitimize public interventions in the agricultural sector. The main obstacle of this public intervention is to know the goods and services provided by agricultural systems and elicitation of the social preferences for them. We created a descriptive approach for the multifunctionality of agricultural systems that is based on the review of the scientific literature focused on multifunctionality and the goods and services of agricultural systems. The review shows a large variety of activities and approaches, which can be grouped by their economic dimension (E), social dimension (S) and environmental dimension (EN). Multicriteria techniques, such as the Analytic Hierarchy Process (AHP), can help elicit the priorities and the relative importance of different functions attributed by the society as a whole. The authorities can take into account these results to inform and support their political decisions. This paper describes a methodological approach to determine the Social Welfare Function (SWF) by using AHP. The proposed methodology is applied to the Huerta de Valencia, a rich peri-urban agricultural system with a variety of resources, around which there is an open political-institutional debate to define a protection scheme. The results are very interesting and useful to enrich this debate.
\end{abstract}

Keywords: Muntifunctional agriculture, public goods, joint production, externalities, market failure, optimal social, multicriteria

\section{Introducción}

Existen determinados sistemas agrarios en entornos periurbanos que se caracterizan por ser espacios de gran valor, a los que se les reconoce el desempeño de funciones que resultan en la provisión de bienes y servicios públicos para los que existe una demanda creciente en la sociedad, como los relacionados con el ocio y la recreación, la producción de alimentos sanos y seguros, la preservación del patrimonio natural y cultural, la conservación del medio ambiente, etc.; en general se considera una oportunidad para estos sistemas agrarios cambiar el modelo de producción tradicional enfocado al mercado, reemplazándolo por un nuevo sistema productivo orientado al consumidor en sentido más amplio. 
Los bienes y servicios públicos que generan los sistemas agrarios son simultáneos a la producción de alimentos y materias primas, pero a diferencia de estos carecen de mercado en los que el agricultor pueda intercambiarlos por rentas; por otra parte, muchos de los bienes públicos asociados a las actividades agrarias tienen un carácter fuertemente local (Reig, 2002). El reconocimiento del fallo de mercado justifica la intervención pública, en aras de la conservación y preservación de estos espacios agrarios.

Para definir la intervención se requiere, en primer lugar, el análisis funcional de los sistemas agrarios para conocer las funciones y los bienes y servicios no comerciales que pueden proveer. En este sentido el concepto de multifuncionalidad agraria constituye un marco teórico muy valioso para caracterizar los sistemas agrarios. En segundo lugar debe ser estimada la demanda social de estos bienes y servicios, para poder integrarla en las decisiones políticas y promover aquellas funciones de los sistemas agrarios con los que se maximizaría la utilidad social de los mismos. En los últimos años son frecuentes los trabajos de investigación que recurren a la utilización de la metodología Analytic Hierarchy Process (AHP) para la determinación de las preferencias sociales por los bienes y servicios públicos que la agricultura y el medio ambiente pueden proveer (Kallas y otros (2007). Esta metodología ha sido utilizada en gestión forestal (Ríos et al., 1998; Nordström et al., 2010; Maroto et al., 2013), impacto ambiental (Ramanathan, 2001), gestión de recursos hídricos (Mesa et al., 2008), explotación de humedales (Blaeij et al., 2009), y en valoración de bienes y servicios ambientales (Aznar \& Estruch, 2007). AHP también ha sido utilizado en estudios agrarios para determinar preferencias sociales por áreas rurales (Duke \& Aull-Hyde, 2002), por áreas rurales de regadío (Gómez-Limón, 2006), por los sistemas alternativos de cultivo del olivar en España, (Parra-López et al., 2008), ola aceptación social de la acuicultura, en concreto de la industria del salmón, en Escocia (Whitmarsh \& Palmieri, 2009).

En este trabajo presentamos un enfoque metodológico para la aplicación de la metodología AHP al estudio de las preferencias sociales por las distintas funciones que pueden desempeñar los sistemas agrarios, en particular la modelización para definir una jerarquía más coherente, la recopilación de los juicios de valor a través de cuestionarios adecuadamente diseñados, y la priorización y la sistemática de agregación de las preferencias sociales que permita determinar la Función de Utilidad Social (FUS), base para la implementación de políticas de conservación y preservación de los sistemas agrarios. La propuesta metodológica se aplica a la Huerta de Valencia, sistema agrario de gran riqueza y con gran variedad de recursos, sobre el que hay abierto un debate político-institucional para definir un régimen de protección. 


\section{Materiales y métodos}

La Huerta de Valencia es un sistema agrario de gran riqueza y gran variedad de recursos, que está sujeto, por otra parte, a presiones que pueden condicionar su evolución y su pervivencia. Por su carácter de agricultura periurbana, pues se ubica en el Área Metropolitana de la ciudad de Valencia, ha estado muy condicionada por este hecho y las fuertes presiones por la competencia de actividades alternativas en este espacio. Recientemente ha sido objeto de protección a través de una figura legal recogida en la normativa valenciana, el Plan de Acción Territorial de Protección de la Huerta.

En la tabla adjunta se concretan los resultados del análisis previo detallado de la multifuncionalidad de este sistema agrario, diferenciando los que tienen carácter privado de los que tienen carácter público, y entre los que tienen carácter público los que constituyen externalidades positivas de los que constituyen externalidades negativas. Para el análisis se propusieron una serie de indicadores, a partir de cuyos valores se constata la intensidad o grado en que se manifiesta cada externalidad, estableciendo seis niveles (+ Favorable, ++ Bastante favorable, +++ Muy favorable, - Desfavorable, -- Bastante desfavorable, --- Muy desfavorable), mostrando la situación actual (SA) para cada una de las funciones y de los bienes y servicios.

De acuerdo con la literatura referente a la utilización del AHP (Proceso Analítico Jerárquico) se observa, que si bien esta metodología fue diseñada por Thomas Saaty para su utilización en procesos de toma de decisiones a nivel individual, o de grupos pequeños de expertos, desde hace ya algunos años, son muchos los autores que la han utilizado como metodología de valoración multicriterio en la determinación de las preferencias sociales por determinados bienes o servicios.

El AHP consiste en establecer una estructura jerárquica del problema de toma de decisión, con el objetivo de presentar toda la información, descomponerla, y ordenarla para poder llevar a cabo el proceso de decisión. En los últimos años se han propuesto diferentes actualizaciones del método AHP: En su trabajo de 2005, Saaty propone una generalización del método AHP, el Proceso de Análisis de Redes (Analytic Network Process o ANP), que permite tener en cuenta la interdependencia entre los niveles del problema de decisión.

La metodología AHP es relevante en el presente contexto en la medida en que las funciones que desempeñan los sistemas agrarios presentan diversas dimensiones (económica, social y medioambiental) y esta metodología provee una manera de medir su demanda en una escala no monetaria. La aplicación de esta metodología en el análisis de preferencias sociales permite un resultado que se puede interpretar y validar en términos de funciones de utilidad social, valorando, en el caso que nos ocupa, las prioridades de los distintos colectivos sociales afectados, mediante el análisis de la importancia relativa que el conjunto de la sociedad otorga a las diferentes funciones (Duke\&Aull-Hyde, 2002; Hall et al., 2004; Reig, 2007). 
Tabla 1. Funciones, bienes y servicios de la Huerta de Valencia

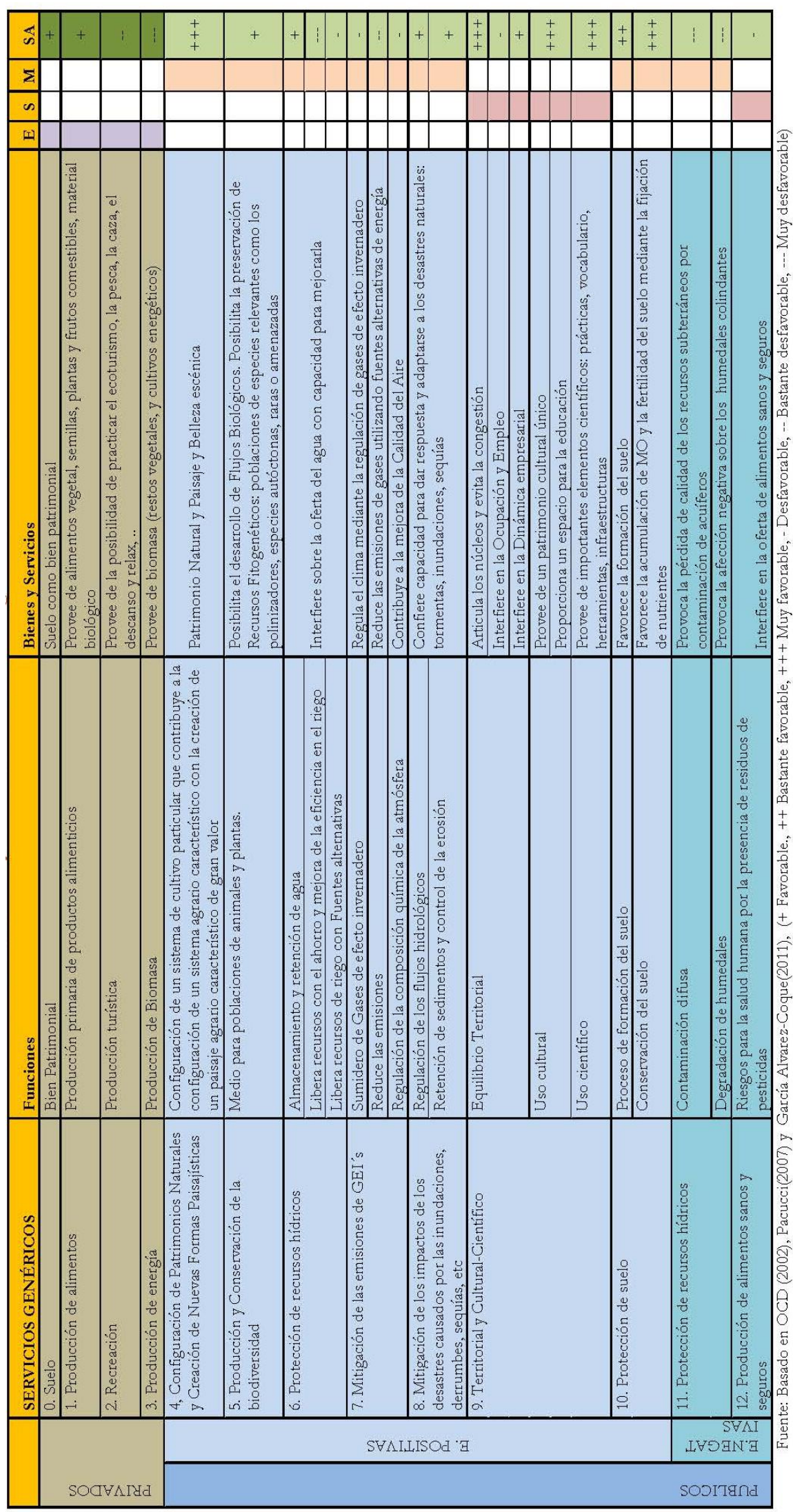


El AHP se fundamenta en varias etapas. Cuatro fundamentalmente:

\section{a) Modelización: formulación del problema de decisión en una estructura jerárquica.}

Consiste en el establecimiento de una representación jerárquica del problema, que incluya todos los aspectos relevantes del problema de decisión. En el análisis de los sistemas agrarios, el objetivo principal podría ser: maximizar el bienestar social, maximizar la producción de bienes y servicios, etc. Los trabajos de investigación orientados a la determinación de las preferencias de la sociedad por los sistemas agrarios en aras de su conservación y preservación, se pueden desarrollar a partir de la funcionalidad determinada para el sistema agrario en cuestión, y estudiando las preferencias sociales por las distintas funciones que estos pueden desempeñar. La aplicación del AHP busca la determinación de la función de utilidad, y por ello, en nuestro trabajo, el objetivo general es la maximización de la utilidad de la Huerta.

De acuerdo con este objetivo general, el segundo nivel de la jerarquía está representado por los criterios y subcriterios de decisión, y que constituyen los aspectos que determinan la funcionalidad de los sistemas agrarios. Seguiremos para ello el esquema propuesto en el análisis de la funcionalidad de los sistemas agrarios, diferenciando las funciones que pueden proveer, por su carácter económico, social y medioambiental. Coincide este esquema, con los planteamientos generales propuestos por Saaty (1997), en la aplicación del proceso analítico-jerárquico en la toma de decisiones de política pública.

La composición de la función de utilidad social así obtenida, refleja la importancia relativa que la sociedad otorga a las distintas funciones que pueden desarrollar los sistemas agrarios. En la medida que la ponderación de atributos con fines valorativos debe hacerla el conjunto de la sociedad analizada, debe realizarse sobre una muestra representativa de la sociedad.

De acuerdo con el esquema propuesto para el análisis de la funcionalidad del sistema agrario de la Huerta de Valencia hemos planteado la correspondiente jerarquía, que incluye las distintas funciones que puede desarrollar este sistema agrario, agrupadas según su carácter económico, social o medioambiental. A partir del análisis funcional, se ha realizado una serie de consideraciones tendentes a la obtención de una jerarquía coherente, con un número limitado de criterios y subcriterios, agrupándolos y concretándolos, de manera que se aporte la información necesaria y suficiente, que permita una mejor consistencia. Por otra parte, todos los criterios y subcriterios, se orientan en el proceso de decisión al más mejor, reconvirtiendo las externalidades negativas en funciones deseables: oferta de recursos hídricos de calidad y oferta de alimentos sanos y seguros. La agregación de los diferentes subcriterios, en nuestro caso funciones, se ha realizado para aquellas con idéntico carácter y que pueden abarcarse de forma conjunta, evitando la información excesiva para los decisores, que en caso contrario tienden a ignorar determinados objetivos menos importantes y a tratar incoherentemente el resto. Podría procederse también a la eliminación de objetivos irrelevantes, pero no es el caso, se ha optado mejor por la agrupación. La composición de la función de utilidad social así obtenida, refleja la importancia relativa que la sociedad otorga a las distintas funciones que pueden desarrollar los sistemas agrarios. 


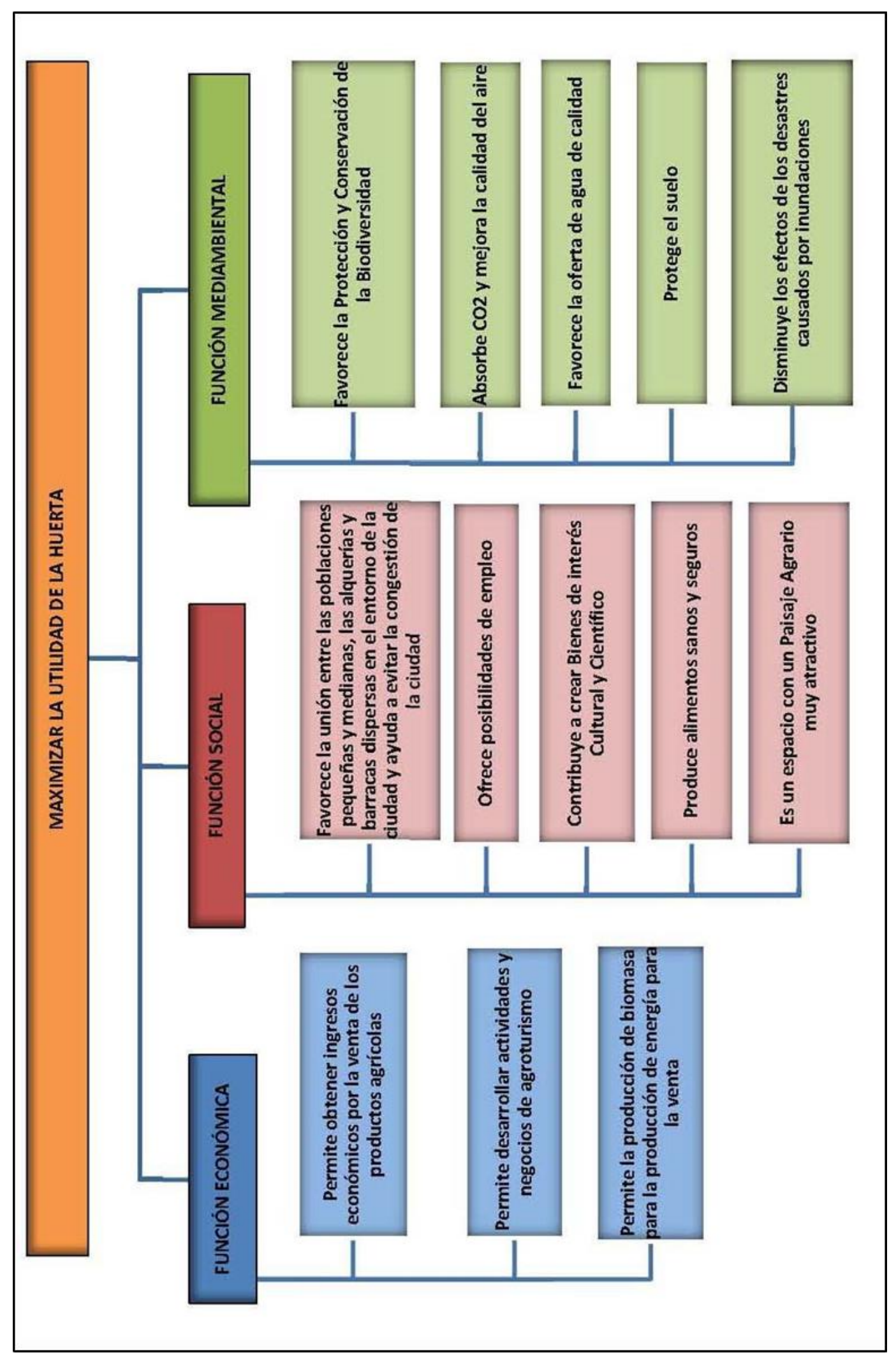

Fig. 1. Planteamiento de la jerarquía 
Para el estudio de las preferencias por las funciones de la Huerta, se ha definido cómo ámbito geográfico, la ciudad de Valencia y su área metropolitana. La ficha técnica de la encuesta se muestra en la Tabla 2 adjunta.

La distribución de los cuestionarios se realizó teniendo en cuenta aspectos demográficos y socioeconómicos de la población. Se distribuyeron por poblaciones según el número de habitantes, y se distribuyeron por sexo y edad, en todos los casos, según los datos del Padrón Municipal de 2011 publicados por el IVE.

Tabla 2. Ficha Técnica Encuesta Ciudadana

\begin{tabular}{|c|c|}
\hline Población objeto de estudio & Personas residentes en Valencia y Área Metropolitana \\
\hline & 1.544.729 Hab \\
\hline Universo & Personas de ambos sexos, mayores de 18 años \\
\hline Tamaño de muestra & 410 individuos \\
\hline Tipo de entrevista & $\begin{array}{l}\text { Los cuestionarios se han aplicado mediante entrevista personal en } \\
\text { domicilios o en la calle. }\end{array}$ \\
\hline Puntos de muestreo & 1 provincia, 25 municipios \\
\hline Tipo de muestreo & $\begin{array}{l}\text { Polietápico, estratificado por conglomerados (afijación proporcional } \\
\text { al tamaño del hábitat), con selección de las unidades primarias de } \\
\text { muestreo (municipios) y de las unidades últimas de muestreo (indivi- } \\
\text { duos) por rutas aleatorias y cuotas de sexo y edad (intervalos:18-25 } \\
\text { años, } 26-40 \text { años, } 41-65 \text { años, y mayores de } 65 \text { años). }\end{array}$ \\
\hline Error & Error muestral inferior al 5\% \\
\hline Fecha de trabajo de campo & 14 de Noviembre a 19 de Diciembre de 2012 \\
\hline
\end{tabular}

b) Valoración: los distintos participantes emiten sus juicios mediante comparaciones pareadas entre los elementos del problema en cuestión.

Una vez constituida la jerarquía, y definida la muestra representativa, se procede a la consulta, para que emitan sus juicios de valor o preferencias en cada nivel jerárquico. La consulta sobre las preferencias se realizó a través de una encuesta. De forma genérica, en la aplicación de la técnica AHP, para emitir sus juicios de valor, cada decisor realiza comparaciones subjetivas por pares (comparaciones binarias). El decisor emite juicios de valor sobre la importancia relativa que otorga a los criterios y alternativas, de forma que quede reflejado la dominación relativa, en términos de importancia, preferencia o probabilidad, de uno frente otro, respecto de los criterios que se comparan.

Las comparaciones se realizan por pares dentro de cada nivel de la jerarquía definida. En el esquema propuesto sobre la funcionalidad de los sistemas agrarios, inicialmente se comparan las funciones de carácter genérico: económica, social y medioambiental. Posteriormente se comparan las correspondientes al tercer nivel, comparando entre sí las funciones explicativas de cada una de las funciones genéricas, de manera que sólo se comparan entre sí, las funciones que cuelgan de un mismo nodo. 
Cada decisor debe emitir $\mathrm{Nx}(\mathrm{N}-1) / 2$ juicios de valor sobre la importancia relativa de $\mathrm{N}$ criterios, generando su matriz de Saaty $\mathrm{A}$, donde $a_{i j}$, representan los resultados obtenidos de comparar el criterio i con el criterio j. $a_{i j}$ tomará valores entre 9 y $1 / 9$. Se denomina matriz de preferencias o de comparaciones pareadas.

El AHP permite al decisor realizar las comparaciones y mostrar no sólo su preferencia, sino también cuantificar la intensidad con que prefiere una función frente a otra con la que se compara, a través de la escala de medida propuesta por Saaty, que queda recogida en la Tabla 3 adjunta.

Tabla 3 Escala de comparaciones

\begin{tabular}{|l|l|c|}
\hline \multicolumn{2}{|c|}{ ESCALA DE COMPARACIONES } \\
\hline Igual Importancia & $\mathbf{1}$ & $=$ \\
\hline Importancia intermedia entre 1y 3 & 2 & \\
\hline Ligeramentemásimportante & $\mathbf{3}$ & + \\
\hline Importancia intermedia entre 3 y 5 & 4 & \\
\hline Másimportante & $\mathbf{5}$ & ++ \\
\hline Importancia intermedia entre 5 y 7 & 6 & \\
\hline Evidentementemásimportante & $\mathbf{7}$ & +++ \\
\hline \hline Importancia intermedia entre 7 y 9 & 8 & \\
\hline Extremadamentemásimportante & $\mathbf{9}$ & ++++ \\
\hline
\end{tabular}

Los encuestados fueron entrevistados para revelar su preferencia por las funciones que puede desempeñar el sistema agrario de la Huerta, a través de una empresa espe-cializada en el desarrollo de estudios de carácter social.

c) Priorización: se obtienen las prioridades, a partir de alguno de los métodos de priorización existentes (vector principal)

A partir de las matrices de preferencias individuales se calculan las prioridades de cada individuo respecto de las distintas funciones. El cálculo de las prioridades se realizó siguiendo el Método del Vector Principal (EM). Aunque existen otros métodos alternativos para el cálculo de las prioridades, se optó por el EM, porque que es el método propuesto por Saaty, y porque es uno de los pocos métodos que también provee un índice para comprobar la consistencia de los juicios emitidos por los encuestados.

El siguiente paso es obtener los pesos representativos del conjunto de los encuestados, que a su vez representan a la sociedad del área de estudio, y determinar la FUS. 
Una primera cuestión a resolver, es como procedemos a agregar los juicios individuales, para obtener la Función Social. En este sentido, se puede proceder a la agregación primero, de las preferencias individuales para obtener las preferencias totales de la sociedad, y a partir de estas las prioridades, o bien, se procede calculando primero las prioridades individuales, que serán las que se agregan para obtener las prioridades del conjunto. Como norma generalmente aceptada por la literatura, la agregación de prioridades individuales se realiza cuando los individuos participan como individuos separados, y la agregación de juicios de preferencias, cuando los individuos forman un grupo más o menos homogéneo.

Forman y otros (1998) consideran que cuando los entrevistados actúan en su propio derecho, con diferentes sistemas de valores, debemos preocuparnos por las prioridades resultantes de cada uno de ellos, y agregar las prioridades individuales, en lugar de las preferencias. Basándonos en estas recomendaciones, en el cálculo de las prioridades sociales a partir de los integrantes de la muestra, deberíamos proceder con el método de agregación de las prioridades individuales (AIP), para al cálculo de la FUS. La agregación se realizó por el método de la media geométrica propuesto por Aczel y otros (1983), Easley (2000), Saaty y otros (2005), Aull-Hyde y otros (2006).

\section{d) Sintesis: obtención de las prioridades totales.}

Calculadas las prioridades individuales se procedido a la agregación de las prioridades de los 404 encuestados de la muestra que presentaban las cuatro matrices completas, para determinar la Función de Utilidad.

Además de las cuestiones relativas a las preferencias, en el cuestionario se incluían también cuestiones para la caracterización socioeconómica de los que han participado en el estudio de preferencias. A partir de las variables de carácter socioeconómi$\mathrm{co}$, hemos estudiado las preferencias sociales por las funciones que puede desempeñar la Huerta, y las funciones de utilidad de los distintos grupos sociales definidos de acuerdo con las características socioeconómicas de los encuestados.

\section{Resultados}

La Huerta de Valencia, es un sistema agrario de gran riqueza y con gran variedad de recursos, sobre el que hay abierto un debate político-institucional para definir un régimen de protección. Con esta investigación hemos pretendido, constatar, que existe una preocupación e interés social en la Huerta, y en su conservación y preservación. De hecho, en los resultados de la encuesta que se incluye en el presente trabajo de investigación, se ha comprobado que el 65\% de los encuestados, que constituyen una muestra representativa de la población de Valencia y su área metropolitana, se muestran interesado en el estado y conservación de este espacio agrario. 
Tabla 4. "Como se consideran" los encuestados con relación a la Huerta

\begin{tabular}{|l|c|c|}
\hline \multicolumn{1}{|c|}{ COMO SE CONSIDERA } & $\mathbf{N}^{\circ}$ INDIVIDUOS & $\%$ \\
\hline Muy interesado & 33 & $8,17 \%$ \\
\hline Bastante interesado & 124 & $30,69 \%$ \\
\hline Algo interesado & 105 & $25,99 \%$ \\
\hline Poco interesado & 49 & $12,13 \%$ \\
\hline Nada interesado & 23 & $5,69 \%$ \\
\hline Sin especificar & 70 & $17,33 \%$ \\
\hline
\end{tabular}

Fuente: encuesta trabajo de investigación.

Este interés social se concreta en las preferencias sociales por las funciones y los bienes y servicios que puede generar este espacio agrario, constatándose la utilidad de la metodología AHP como instrumento útil para la determinación de las preferencias sociales y su interpretación en términos de utilidad, y de demanda social.

- la función económica es la de mayor importancia: 38,5\%.La preferencia por la función medioambiental se sitúa en el entorno del 34\%, y la función social es la menos importante con un peso del $27,46 \%$.

- la función de generar rentas agrarias es la más importante entre las económicas, y también sobre el resto de explicativas, con un peso superior al $20 \%$. Mientras al resto de funciones explicativas, se le asigna una importancia inferior al 10\%. Siendo otras funciones explicativas importantes con pesos en torno al 7\%: turismo, ocupación y empleo, alimentos sanos y seguros, y absorción de CO2.

Tabla 5: Función de Utilidad Social

\begin{tabular}{|c|c|c|}
\hline & & $\begin{array}{c}\text { FUNCIÓN DE } \\
\text { UTILIDAD }\end{array}$ \\
\hline \multirow[t]{4}{*}{$\mathrm{wE}$} & & $38,46 \%$ \\
\hline & wRA & $24,13 \%$ \\
\hline & wT & $7,29 \%$ \\
\hline & wEng & $7,05 \%$ \\
\hline \multirow[t]{6}{*}{ wS } & & $27,46 \%$ \\
\hline & wART & $3,96 \%$ \\
\hline & wCOP & $7,12 \%$ \\
\hline & wPATR & $4,80 \%$ \\
\hline & wALI & $7,39 \%$ \\
\hline & wPA & $4,18 \%$ \\
\hline wMA & & $34,08 \%$ \\
\hline \multirow{5}{*}{ WNIA } & wBIOdv & $6,36 \%$ \\
\hline & wAbscO2 & $8,41 \%$ \\
\hline & wOfqH2O & $6,85 \%$ \\
\hline & wPrtSlo & $7,42 \%$ \\
\hline & wPrtInun & $5,02 \%$ \\
\hline
\end{tabular}


La inclusión en el cuestionario de datos de carácter socioeconómico, ha permitido definir grupos de encuestados de acuerdo con estas características socioeconómicas, y estudiar sus funciones de utilidad. Observándose diferencias en la Función de Utilidad Social calculada, según el sexo, la edad, el nivel de estudios, y según que los encuestados residan en la ciudad de Valencia o en su área metropolitana, y el interés manifestado por los individuos, por la Huerta. Los resultados más interesantes de cara a establecer conclusiones sobre preferencias de la población, son:

A. Por sexos, se observa que tanto para hombres como para mujeres, la función económica es la más importante, y con un peso similar al del promedio de la población en su conjunto. Por otra parte, las mujeres otorgan una mayor relevancia a la función social, y dentro de esta, a la función de contribuir a la ocupación y el empleo, mientras que la función medioambiental la consideran menos importante, a diferencia de los hombres, que sin embargo, otorgan una mayor importancia a la función medioambiental frente a la social.

B. Por edades, los grupos 18-25 y 41-65, no muestra diferencias respecto a la media de la muestra. Las diferencias se observan en el grupo de 25-40, para los que la función medioambiental de la Huerta es la más importante, y dentro de esta la conservación de la biodiversidad, la absorción de CO2 y la protección del suelo, si bien la función de rentas sigue siendo muy importante.

C. Según el nivel de rentas, se observa gran disparidad en las preferencias de las funciones, entre los distintos grupos de nivel de renta y también con relación a los valores de preferencias que manifiesta en promedio la muestra de la población.

D. Los ciudadanos con mayor nivel de estudios otorgan mucha menor importancia a la función económica, en favor de la función medioambiental y social.

E. Los habitantes del Área metropolitana otorgan una mayor importancia a la función económica y también ligeramente mayor es la importancia que otorgan a la Huerta como generadora de rentas agrarias. Mientras que los habitantes de la ciudad de Valencia muestran unos pesos muy similares a los del total de los individuos de la muestra.

F. Un dato interesante obtenido de la muestra, es el interés en la Huerta que manifiestan los entrevistados. En este sentido, como ya se indicó con anterioridad, y puede comprobarse en la Tabla 4, de los 404 individuos, 262, el 65\% de los encuestados, se muestra más o menos interesado en la Huerta, mientras que el $35 \%$ restante se considera poco o nada interesado en la Huerta. Cuanto mayor es el interés que muestra los individuos por la Huerta, menor es la importancia que otorgan a la función económica, y menor la importancia como generadora de rentas agrarias, otorgando un mayor peso a la función medioambiental y social, y dentro de estas, mayor importancia a las funciones de protección del patrimonio, del paisaje agrario, y la biodiversidad. Mientras que los individuos que se manifiestan poco interesados en la Huerta, se muestran totalmente contrarios, en sus prioridades, a los anteriores. Consideran la función económica muy importante, y la actividad agraria como la gran contribuidora a la generación de rentas económicas, mientras que a las funciones de protección y conservación del patrimonio cultural, y del paisaje agrario no les otorgan prácticamente importancia. 


\section{Conclusiones}

Esta investigación pretende contribuir al reconocimiento de la multifuncionalidad de la agricultura, defendiendo una actividad que desempeña una función económica, a la vez que otras funciones relativas la protección del medio ambiente y del paisaje, a la conservación del patrimonio cultural, a la mejora de los aspectos de equilibrio social, así como la posibilidad de proveer servicios recreativos. Estas funciones generan bienes y servicios que no tienen mercado, pero para los que existe una demanda social.

El trabajo de investigación parte de un desarrollo previo a partir del cual se concretó una aproximación descriptiva de la multifuncionalidad de la agricultura, que es la base para el análisis de la demanda de los bienes y servicios que pueden proveer los sistemas agrarios, y las funciones que pueden desempeñar, así como para la determinación de una función de utilidad, que guíe la definición de las políticas de intervención. El esquema se ha aplicado al caso concreto de la Huerta de Valencia, que es un sistema agrario de gran riqueza y con gran variedad de recursos, sobre el que hay abierto un debate político-institucional para definir un régimen de protección. La estimación de las preferencias sociales por las funciones que desarrolla la Huerta, se ha realizado mediante metodologías de análisis multicriterio, y más concretamente el Analytic Hierarchy Process (AHP) para valorar las prioridades sociales e interpretarlas en términos de utilidad, y de demanda social, para integrarlas en las decisiones de políticas encaminadas a la promoción de aquellas funciones de los sistemas agrarios con los que se maximizaría la utilidad del mismo.

Los resultados deberían integrarse en el proceso político-institucional de toma de decisiones sobre políticas de intervención, desarrollado actualmente sobre este espacio agrario para definir el régimen de protección.

Agradecimientos. Los autores agradecen el soporte recibido del Ministerio de Economía y Competitividad de España, a través del Proyecto de investigación Técnicas multicriterio y toma de decisiones participativa para una gestion sostenible, Ref. ECO2011-27369. 


\section{Referencias}

Aczel, J., \& Saaty, T. (1983): Procedures for Synthesizing Ratio Judgements. Journal of Mathematical Psychology, 27, 93-102.

Alvarez-Coque, J.M.G\& López-García, T. (2011): Viabilidad del Espacio Agrario Urbano de la Huerta de Valencia. Actes del III Congrés d'Estudis de l'Horta Nord. Valencia.

Aull-Hyde, R., Erdogan, S., \& Duke, J. (2006): An experiment on the consistency of aggregated comparison matrices in AHP. European Journal of Operational Research, 171, 290295.

Aznar, J., \& Estruch, V. (2007): Valoración de activos ambientales mediante métodos multicriterio. Aplicación a la valoración del Parque Natural del Alto Tajo. Economía Agraria y Recursos Naturales, 7(13): 107-125.

De Blaeij, A., Linderhof. V., Polman, N., Reinhard, S., (2009): Social preferences for exploiting commercial wetlands. Conf on Economic Instruments to Enhance the Conservation and Sustainable Use of Biodiversity. Venice (Italia).

Duke, J., Aull-Hyde, R., (2002): Identifying public preferences for land preservation using the analytic hierarchy process. Ecological Economics 42: 131-145.

Easley, R., Valacich, J., \& Venkataramanan, M. (2000): Capturing group preferences in a multicriteria decision. European Journal of Operational Research, 125, 73-83.

Forman, E., \& Peniwati, K. (1998): Aggregating individual judgments and priorities with the Analytic Hierachy Process. European Journal of Operation Research, 9, 108-165.

Gómez-Limón, J. (2006): El regadío: sistema agrario multifuncional. Agricultura Familiar en España, 117-127.

Hall, C., McVittie, A., \& Moran, D. (2004): What does the public want from agriculture and the countryside?A review of evidence and methods. Journal of Rural Studies, 20, 211-225.

Kallas, Z., Gómez-Limón, J., \& Barreiro, J. (2007): Oferta y Demanda de Bienes y Servicios Públicos de la Agricultura Española. En La multifuncionalidad de la agricultura en España (págs. 131-153): MAPA, Eumedia, Madrid.

Maroto, C., Segura, M., Ginestar, C., Uriol, J., Segura, B. (2013): Sustainable Forest Management in a Mediterranean region: social preferences. Forest Systems 22(3): 546-558.

Mesa, P., Martín-Ortega, J., Berbel, J. (2008): Análisis Multicriterio de Preferencias Sociales en Gestión Hídrica bajo la DMA. Economía Agraria y Recursos Naturales 8(2): 105-1026.

Nordström, E-M., Eriksson, L.O., Öhman, K. (2010): Integrating multiple criteria decision analysis in participatory forest planning: Experience from a case study in northern Sweden. Forest Policy and Economics 12: 562-574.

Parra-López, C., Calatrava-Requena, J., \& de Haro-Giménez, T. (2008): A systemic comparative assessment of the multifuntional performance of alternative olive system in Spain within an AHP-extended framework. Ecological-Economics. 
Pascucci, S. (2007): Agricoltura periurbana e strategie di svilippo rurale. Università degli Studi di Napoli Federico II, Dipartamento Di Economia e Politica Agraria, Nápoles.

Ramanathan, R. (2001): A note on the use of the analytic hierarchy process for envionmental impact assessment. Journal of Environmental Management 63: 27-35.

Reig, E. (2002): La Multifuncionalidad del Mundo Rural. Información Comercial Española, 803, $33-44$

Reig, E. (2007): Fundamentos Económicos de la Multifuncionalidad. En P. y. Ministerio de Agricultura, La Multifuncionalidad de la Agricultura Española (págs. 19-39). Madrid: Eumedia.

Ríos, V., Díaz, L., \& Romero, C. (1998): Economía y gestión ambiental: un enfoque decisional multicriterlo. Real Academia de Ciencias Exactas, Físicas y Naturales, 92(4), 399-408.

Saaty, T. (1997): Toma de decisiosnes para líderes: El proceso analítico jerárquico la toma de decisiones en un mundo complejo. RWS Publications.

Saaty, T., \& Vargas, L. (2005): The possibility of group welfare functions. 4, 167-176.

Whitmarsh, D. \& Palmieri, M. (2009): Social acceptability of Marine Aquiculture: The use of survey-based methods for eliciting public and stakeholders preferences. 33: 452-457. 